Pacific Journal of Mathematics

ON THE THEORY AND APPLICATION OF SUM
COMPOSITION OF LATIN SQUARES AND ORTHOGONAl

LATIN SQUARES 


\section{ON THE THEORY AND APPLICATION OF SUM COMPOSITION OF LATIN SQUARES AND ORTHOGONAL LATIN SQUARES}

\section{A. Hedayat and E. Seiden}

The object of this paper is three-fold. First, it puts the theory of "sum composition" of Latin squares and orthogonal Latin squares in its most precise form. Second, it compiles and unifies previous results which have appeared in technical reports and in proceedings of a conference in Italy, which are not readily available. Finally, it presents some new results in this area.

The research relates to the following question: given two Latin squares $L_{1}$ and $L_{2}$ of order $n_{1}$ and $n_{2}\left(n_{1} \geq n_{2}\right)$, respectively, in how many ways (if any at all) can one compose $L_{1}$ and $L_{2}$ in order to obtain a Latin square $L_{3}$ of order $m$, where $m$ is a function of $n_{1}$ and $n_{2}$ only? It is well known that $L_{3}$ $=L_{1} \otimes L_{2}$ is a Latin square of order $n_{1} n_{2}$ irrespective of the combinatorial structures of $L_{1}$ and $L_{2}$. The theory produces a Latin square $L_{3}$ of order $n_{1}$ $+n_{2}$ (thus the name "sum composition"), provided $L_{1}$ has a certain combinatorial structure. Although this method does not work for all pairs of Latin squares, it has an immediate application in the construction of orthogonal Latin squares with certain interesting and useful combinatorial structures, including those of order $4 t+2, t \geq 2$. As will be seen this method is easy, and is simpler than other known methods for the construction of orthogonal Latin squares of order $4 t+2$ (see [1]). Perhaps the idea of sum composition can be extended to other combinatorial structures and designs.

In $\$ 2$ preliminary concepts and definitions are presented, which are then used in the following sections. Section 3 develops the basic idea of the sum composition of Latin squares and points out the usefulness of these concepts. Section 4 introduces the idea of horizontal and vertical projections of a transversal on rows and columns. It also introduces the idea of "capturing" a lost transversal. It contains two lemmas for capturing a transversal by horizontal and vertical projections. These lemmas are considered to be the fundamental lemmas in sum composition. In $\$ 5$ a technique is developed which forms a basis for the construction of an $\mathrm{O}(n, 2)$ via sum composition of an $\mathrm{O}\left(n_{1}, 2\right)$ based on $\mathrm{GF}\left(n_{1}\right)$ and an arbitrary $\mathrm{O}\left(n_{2}\right.$, 2). Section 6 introduces a family of $\mathrm{O}(n, 2)$ with a sub $\mathrm{O}\left(n_{2}, 2\right)$ by sum composition of an $\mathrm{O}\left(n_{1}, 2\right)$ based on $\mathrm{GF}\left(n_{1}\right)$ and an arbitrary $\mathrm{O}\left(n_{2}, 2\right)$ where $n_{2}$ hits the upper bound namely $\left[n_{1} / 2\right]$, where $[a]$ denotes the integer 
part of $a$. Section 7 presents a family of $\mathrm{O}(n, 2)$ with a sub $\mathrm{O}(3,2)$ by sum composition of an $\mathrm{O}\left(n_{1}, 2\right)$ based on $\mathrm{GF}\left(n_{1}\right)$ of odd order and an $\mathrm{O}(3,2)$. The lowest order which $n_{2}$ can take on in the present theory is 3 (also see $\S 10)$. In $\S 8$ a family of $O\left(p^{\alpha}+4,2\right)$ with sub $O(4,2)$ is constructed. Section 9 concerns itself with the construction of a family of $\mathrm{O}\left(p^{\alpha}+5,2\right)$ with sub $O(5,2)$. The order of the Latin squares composed in this case is always of the form $4 t+2$. Section 10 discusses extensions of the theory in two different ways. Several unsolved problems are also stated.

2. Preliminaries. Let $\Sigma$ be a set of cardinality $n \geq 1$. Let $L$ be a Latin square of order $n$ on $\Sigma$.

Definition 2.1. $L$ is said to have a transversal if there exists a collection of $n$ cells in $L$ with the properties that: (i) no row and column of $L$ contains more than one cell of this collection, (ii) the entries of these cells exhaust the set $\Sigma$.

Of course, not every Latin square has a transversal.

Definition 2.2. $L$ is said to have $t$ parallel transversals if $L$ contains $t$ transversals, no two of which have any cell in common.

Definition 2.3. Let $L_{1}, L_{2}, \ldots, L_{r}$ be $r$ Latin squares of order $n$ on $\Sigma$. Then a collection of $\boldsymbol{n}$ cells is said to form a common transversal for these $r$ Latin squares if the collection is a transversal for each of these $r$ Latin squares.

Definition 2.4. A set of $r$ Latin squares of order $n$ on $\Sigma$ is said to contain $t$ parallel common transversals if they have $t$ common transversals which are pairwise parallel.

Hereafter, the symbol $\mathrm{O}(n, r)$ denotes a set of $r$ pairwise orthogonal Latin squares of order $n$. The notation, $L_{1} \perp L_{2}$, indicates that $L_{1}$ is orthogonal to $L_{2}$. It is easy to see that:

LEMMA 2.1. An $O(n, r)$ exists if and only if an $O(n, r-1)$ with $n$ common parallel transversals exists.

LEMMA 2.2. An $O(n, n-1)$ has no common transversal.

EXAMPLe 2.1. Let $\Sigma=\{a, b, c, d\}$. Then the underlined and parenthesized cells form two common parallel transversals for the following $\mathrm{O}(4,2)$. 


$\begin{array}{lllllllll}(a) & b & \underline{c} & d & & (a) & c & \underline{d} & b \\ c & (d) & a & \underline{b} & & b & (d) & c & \underline{a} \\ \underline{d} & c & (b) & a & , & \underline{c} & a & (b) & d \\ b & \underline{a} & d & (c) & & d & \underline{b} & a & (c)\end{array}$

3. Sum composition of Latin squares. In order to make the reading of this paper independent of our previous papers [4], [5], [6], and [8], the sum composition technique for the construction of Latin squares of order $n_{1}+$ $n_{2}$ from Latin squares of orders $n_{1}$ and $n_{2}$ having certain combinatorial structures is reviewed. Sum composition has numerous applications:

(i) It can be used for the construction of Latin squares of order $n_{1}+$ $\mathrm{n}_{2}$ with sub-Latin squares of order $n_{2}$ for all $n_{1}$ and $n_{2} \leq n_{1}$ except for $\left(n_{1}\right.$, $\left.n_{2}\right)=(2,1),(2,2),(6,5)$ and $(6,6)$.

(ii) It has an immediate application for the construction of pairs of orthogonal Latin squares of order $n_{1}+n_{2}$, including those of the form $4 t+$ 2, with sub-orthogonal Latin squares of order $n_{2}$.

(iii) Latin squares and orthogonal Latin squares constructed via sum composition enjoy certain combinatorial properties which are useful for the construction of several useful experimental designs for successive stages (see Hedayat, Parker and Federer [7]).

(iv) Hedayat [3] has utilized this method and has produced a Latin square of order 10 which is orthogonal to its transpose.

(v) Finally, Federer [2] has pointed out several other applications of sum composition.

Consider an $m \times m$ square $B$ with a Latin square $L$ of order $n<m$ in its top left corner. In the sequel the following concepts will be needed:

(i) the vertical projection of a given transversal in $L$ on the $r$ th row $(r$ $>n)$ of $B$ means placing in the $(r, j)$ cell of this row, that element of the given transversal which appears in the $j$ th column of $L, j=1,2, \ldots, n$.

(ii) Similarly, the horizontal projection of a given transversal on the $t$ th column, $t>n$, of $B$ means placing in the $(i, t)$ cell of this column that element of the given transversal which appears in the $i$ th row of $L, i=1,2$, $\ldots, n$.

The following example clarifies the above concepts.

EXAMPLE 3.1. Let $L$ and $B$ be the following squares. 


$$
L=\begin{array}{rrrr}
1 & \underline{2} & 3 \\
2 & 3 & \frac{1}{3} & \text { and } B=
\end{array}
$$

\begin{tabular}{|l|l|l|l|l|l|}
\hline 1 & $\underline{2}$ & 3 & & & \\
\hline 2 & 3 & $\underline{1}$ & & & \\
\hline$\underline{3}$ & 1 & 2 & & & \\
\hline & & & & & \\
\hline & & & & & \\
\hline & & & & & \\
\hline & & & & & \\
\hline
\end{tabular}

The underlined cells form a transversal for $L$. The vertical and horizontal projections of this transversal on the 6th row and 5th column of $B$ produce the following square.

\begin{tabular}{|l|l|l|l|l|l|}
\hline 1 & 2 & 3 & & 2 & \\
\hline 2 & 3 & 1 & & 1 & \\
\hline$\underline{3}$ & 1 & 2 & & 3 & \\
\hline & & & & & \\
\hline & & & & & \\
\hline 3 & 2 & 1 & & & \\
\hline
\end{tabular}

The method of sum composition will be described next. Let $\Sigma_{1}$ and $\Sigma_{2}$ be two non-intersecting sets of cardinalities $n_{1}$ and $n_{2}$, respectively, $n_{1} \geq n_{2}$. Let $L_{1}$ be a Latin square of order $n_{1}$ with $n_{2}$ parallel transversals on $\Sigma_{1}$. Note that this is always possible except for $\left(n_{1}, n_{2}\right)=(2,1),(2,2),(6,5)$, and $(6,6)$. Let $L_{2}$ be a Latin square of order $n_{2}$ on $\Sigma_{2} . L_{2}$ is not required to have any specific combinatorial structure. Let $C_{1}$ be an $m \times m, m=n_{1}+$ $n_{2}$, square containing $L_{1}$ and $L_{2}$ in the following fashion:

$$
C_{1}=\begin{array}{|l|l|}
\hline L_{1} & \\
\hline & L_{2} \\
\hline
\end{array}
$$

Project horizontally and vertically the $n_{2}$ transversals of $L_{1}$ on the last $n_{2}$ columns and rows of $C_{1}$ in any arbitrary manner. Note that there are $n_{2}$ ! choices for the projections on the rows and $n_{2}$ ! choices for the projections on the columns. Call the resulting square $C_{2}$. Now replace the $n_{1}$ entries of each transversal by a fixed element of $\Sigma_{2}$ such that no two transversals are being replaced by the same element of $\Sigma_{2}$. Call the resulting square $L_{3}$. The above process guarantees that $L_{3}$ is a Latin square of order $n_{1}+n_{2}$ on $\Sigma_{1} U$ $\Sigma_{2}$. 
The preceding steps can be elucidated via an example.

EXAmPle 3.2. Let $\Sigma_{1}=\{1,2,3\}, \Sigma_{2}=\{a, b\}$ and

$$
L_{1}=\begin{array}{ccc}
\frac{1}{2} & (2) & 3 \\
(3) & \frac{3}{1} & (\text { I) }
\end{array} \text { and } L_{2}=\begin{array}{ll}
a & b \\
&
\end{array}
$$

Note that the underlined and parenthesized cells form two parallel transversals for $L_{1}$. Then

$C_{1}=$\begin{tabular}{|c|c|c|c|c|}
\hline$\underline{1}$ & $(2)$ & 3 & & \\
\hline 2 & $\underline{3}$ & $(1)$ & & \\
\hline$(3)$ & 1 & $\underline{2}$ & & \\
\hline & & & $a$ & $b$ \\
\hline & & & $b$ & $a$ \\
\hline
\end{tabular}

and a possible choice of

$C_{2}=$\begin{tabular}{|c|c|c|c|c|}
\hline$\underline{1}$ & $(2)$ & 3 & 2 & 1 \\
\hline 2 & $\underline{3}$ & $(1)$ & 1 & 3 \\
\hline$(3)$ & 1 & $\underline{2}$ & 3 & 2 \\
\hline 1 & 3 & 2 & $a$ & $b$ \\
\hline 3 & 2 & 1 & $b$ & $a$ \\
\hline
\end{tabular}

Observe that the underlined transversal has been projected on the fourth row and on the fifth column and the parenthesized transversal has been projected on the fifth row and on the fourth column. Now replace the entries of the underlined transversal by $a$ and the parenthesized ones by $b$ to obtain 


$L_{3}=$\begin{tabular}{|l|l|l|l|l|}
\hline$a$ & $b$ & 3 & 2 & 1 \\
\hline 2 & $a$ & $b$ & 1 & 3 \\
\hline$b$ & 1 & $a$ & 3 & 2 \\
\hline 1 & 3 & 2 & $a$ & $b$ \\
\hline 3 & 2 & 1 & $b$ & $a$ \\
\hline
\end{tabular}

4. Fundamental lemmas in sum composition. Let $\Sigma_{1}=G F\left(n_{1}\right)$. Let $B(x)$ be a square of order $n_{1}$ with $x \alpha_{i}+\alpha_{j}$ in its $(i, j)$ entry, $x \in \operatorname{GF}\left(n_{1}\right), x \neq$ $0, \alpha_{i}, \alpha_{j} \in \mathrm{GF}\left(n_{1}\right)$. It is well known that $B[x]$ is a Latin square of order $n_{1}$ on $\Sigma_{1}$, and moreover,

$$
B(x) \perp B(y), x \neq y \text {. }
$$

In particular $B(1), B(x)$ and $B(y)$ form an $\mathrm{O}\left(n_{1}, 3\right)$. Note that the $n_{1}$ entries in $B(x)$ and $B(y)$ corresponding to the $n_{1}$ entries equal to $k$, in $B(1)$, i.e., $\alpha_{i}+$ $\alpha_{j}=k \in \mathrm{GF}\left(n_{1}\right)$, form a common transversal for $B(x)$ and $B(y)$. Call this transversal $k$. As $k$ runs over all the elements of $\mathrm{GF}\left(n_{1}\right), n_{1}$ common transversals are obtained for $B(x)$ and $B(y)$. Moreover, two common transversals $k$ and $l, k \neq l$, are parallel. Thus $n_{1}$ common parallel transversals in $B(x)$ and $B(y)$ have been located and named.

Consider the following two $n \times n, n=n_{1}+n_{2}, n_{2} \leq n_{1}$ squares
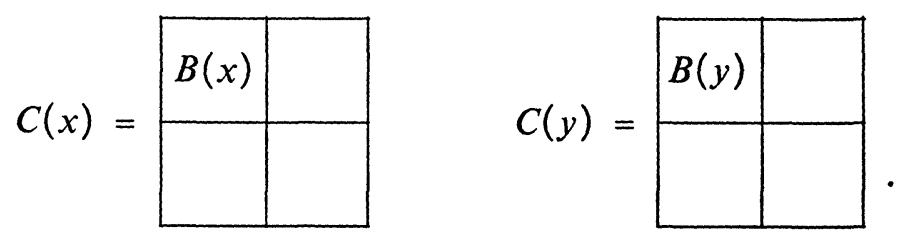

Project the transversal $s$ in $B(x)$ vertically and horizontally on an arbitrary row and column of $C(x)$. Call the resulting square $C^{\prime}(x)$. Also project the transversal $t$ in $B(y)$ vertically and horizontally on the same row and column numbers of $C(y)$. Call the resulting square $C^{\prime}(y)$. The following two lemmas characterize the $2 n_{1}$ ordered pairs obtained upon superposition of $C^{\prime}(x)$ on $C^{\prime}(y)$ corresponding to the projected transversals $s$ and $t$.

LEMMA 4.1. The set of $n_{1}$ ordered pairs resulted from the superposition of the vertical projection of the transversal s in $B(x)$ and transversal $t$ in $B(y)$ forms the same set of ordered pairs as obtained by superposition of the transversal $k_{v}(x, y, s, t)$ in $B(x)$ and in $B(y)$ for 


$$
k_{v}(x, y, s, t)=[s x(1-y)-t y(1-x)] /(x-y), x \neq y .
$$

Proof. The entries of the transversal $s$ in $B(x)$ and the transversal $t$ in $B(y)$ respectively read

$$
\begin{aligned}
& x \alpha_{i}+\alpha_{j}, \alpha_{i}+\alpha_{j}=s \\
& y \alpha_{i}^{*}+\alpha_{j}, \alpha_{i}^{*}+\alpha_{j}=t .
\end{aligned}
$$

Upon vertical projection of these transversals the $n_{1}$ entries respectively read as

$$
x\left(s-\alpha_{j}\right)+\alpha_{j} \text { and } y\left(t-\alpha_{j}\right)+\alpha_{j} .
$$

Therefore upon superposition of these projected transversals the following $n_{1}$ pairs are obtained

$$
\left(x\left(s-\alpha_{j}\right)+\alpha_{j}, y\left(t-\alpha_{j}\right)+\alpha_{j}\right), j=1,2, \ldots, n_{1} .
$$

Now let $\alpha_{i}^{\prime}$ and $\alpha_{j}^{\prime}$ be such that

$$
k_{v}(x, y, s, t)=\alpha_{i}^{\prime}+\alpha_{j}^{\prime}
$$

Upon superposition of transversal $k_{v}(x, y, s, t)$ in $B(x)$ and $B(y)$ one obtains the following $n_{1}$ pairs

$$
\left(x \alpha_{i}^{\prime}+\alpha_{j}^{\prime}, y \alpha_{i}^{\prime}+\alpha_{j}^{\prime}\right)
$$

Equating (4.2) to (4.3) results in

$$
\begin{aligned}
& x\left(s-\alpha_{j}\right)+\alpha_{j}=x \alpha_{i}^{\prime}+\alpha_{j}^{\prime} \\
& y\left(t-\alpha_{j}\right)+\alpha_{j}=y \alpha_{i}^{\prime}+\alpha_{j}^{\prime}
\end{aligned}
$$

which yields the following solution for $k_{v}(x, y, s, t)$.

$$
k_{v}(x, y, s, t)=[s x(1-y)-\operatorname{ty}(1-x)] /(x-y)
$$

Note that if in particular $x=y^{-1}$ the following simple expression for $k_{v}\left(y^{-1}, y, s, t\right)$ holds: 


$$
k_{v}\left(y^{-1}, y, s, t\right)=(t y+s) /(1+y),
$$

which will be denoted by $k_{v}(y, s, t)$ for simplicity.

LEMMA 4.2. The set of $n_{1}$ ordered pairs resulted from the superposition of the horizontal projection of the transversal $s$ in $B(x)$ and transversal $t$ in $B(y)$ forms the same set of ordered pairs as obtained by superposition of the transversal $k_{h}(x, y, s, t)$ in $B(x)$ and in $B(y)$ for

$$
k_{h}(x, y, s, t)=[t(x-1)-s(y-1)] /(x-y), x \neq y .
$$

The proof is analogous to the proof of Lemma 4.1.

If, in particular, $x=y^{-1}$ the expression (4.5) reduces to the following simple expression

$$
k_{h}\left(y^{-1}, y, s, t\right)=(s y+t) /(1+y),
$$

which will be denoted by $k_{h}(y, s, t)$ for simplicity.

REMARK 4.1.

$$
\begin{gathered}
k_{v}(x, y, s, t)+k_{h}(x, y, s, t)=(t+s)+\frac{(t-s)(x y-1)}{(x-y)}= \\
s+t \quad \text { if } x=y^{-1}
\end{gathered}
$$

Remark 4.2. To simplify the detailed descriptions of Lemmas 4.1 and 4.2, they are referred to in the following forms:

(i) The vertical projection of the transversal $s$ in $B(x)$ and the transversal $t$ in $B(y)$ will jointly capture the transversal $k_{v}(x, y, s, t)$ as given in (4.1).

(ii) The horizontal projection of the transversal $s$ in $B(x)$ and the transversal $t$ in $B(y)$ will jointly capture the transversal $k_{h}(x, y, s, t)$ as given in (4.5).

5. An application of sum composition for the construction of sets of orthogonal Latin squares. In order to construct an $\mathrm{O}(n, 2)$ for $n=n_{1}+$ $n_{2}$, we require that $n_{1} \geq 2 n_{2}$ and there should exist an $\mathrm{O}\left(n_{2}, 2\right)$ and an $\mathrm{O}\left(n_{1}\right.$, 2) with $2 n_{2}$ common parallel transversals. In this section, due to some combinatorial difficulties, the case $n_{2}=1$ is excluded even though an $O(1$, 2) exists. The above requirements eliminate the arbitrary decomposition of $n$ into $n_{1}$ and $n_{2}$, for instance, exclude $n_{2}=2$ or 6 . Thus the range of $n_{2}$ is $3 \leq$ 
$n_{2} \leq\left[n_{1} / 2\right]$. The following lemma guarantees that for any $n \geq 10$ there is at least one decomposition of $n$ which fulfills the preceding requirements.

LEMMA 5.1. For any $n \geq 10$ there exists a decomposition $n=n_{1}+n_{2}$ with the property that the existence of an $\mathrm{O}\left(n_{2}, 2\right)$ and an $\mathrm{O}\left(n_{1}, 2\right)$ with at least $2 n_{2}$ common parallel transversals is guaranteed.

Proof. It is a well known fact in number theory that any $n \geq 10$ can be decomposed into $n_{1}+n_{2}, n_{1}=p^{\alpha}, p$ a prime and $\alpha$ a positive integer, $n_{1}$ $\geq 2 n_{2}, n_{2} \geq 3, n_{2} \neq 6$. It is also well known that for any $n_{1}=p^{\alpha}$ there is an $\mathrm{O}\left(n_{1}, n_{1}-1\right)$. These together with the fact that for any $n_{2} \neq 2,6$ there is an $\mathrm{O}\left(n_{2}, 2\right)$, complete the proof.

Now let $n=n_{1}+n_{2}, n \geq 10, n_{1}=p^{\alpha}, n_{2} \geq 3, n_{2} \neq 6$ and $n_{1} \geq 2 n_{2}$. Let $B(x)$ and $B(y), x \neq 1, y \neq 1, x \neq y$, be two Latin squares of order $n_{1}$ on $\Sigma_{1}$ $=\mathrm{GF}\left(n_{1}\right)$. Also let $\left\{A_{1}, A_{2}\right\}$ be an $\mathrm{O}\left(n_{2}, 2\right)$ on $\Sigma_{2}$ of cardinality $n_{2}$ such that $\Sigma_{1} \cap \Sigma_{2}=\phi$. Let $\Omega$ be a set of $2 n_{2}$ parallel transversals for $\{B(x)$, $B(y)\}$. Note that $\Omega$ can be constructed in $\left(\begin{array}{c}n_{1} \\ 2 n_{1}\end{array}\right)$ different ways. Decompose $\Omega$ into two nonintersecting sets $S$ and $T$ each of cardinality $n_{2}$. Let $L_{1}$ be any Latin square of order $n_{1}+n_{2}$ constructed by sum composition of $B(x)$ and $A_{1}$, using the transversals in $S$ (see $\S 3$ ). Let $L_{2}$ be any Latin square of order $n_{1}+n_{2}$ constructed by sum composition of $B(y)$ and $A_{2}$, using the transversals in $T$. The following lemma constitutes the backbone of the remainder of this section.

LeMmA 5.2. $\left\{L_{1}, L_{2}\right\}$ is an $\mathrm{O}(n, 2)$ if $K_{v} \cup K_{h}=\Omega$, where $K_{v}$ and $K_{h}$ denote the sets of captured transversals on rows and columns respectively.

Proof. Upon superposition of $L_{1}$ on $L_{2}$ the following is true:

(i) Every element of $\Sigma_{2}$ in $L_{1}$ appears with every other element of $\Sigma_{2}$ in $L_{2}$, due to the fact that $A_{1} \perp A_{2}$ in the lower right corner.

(ii) Every element of $\Sigma_{2}$ in $L_{1}$ appears with every element of $\Sigma_{1}$ in $L_{2}$ because the entries of the transversals in $S$ have been replaced by the elements of $\Sigma_{2}$.

(iii) Every element of $\Sigma_{1}$ in $L_{1}$ appears with every element of $\Sigma_{2}$ in $L_{2}$ because the entries of the transversals in $T$ have been replaced by the elements of $\Sigma_{2}$.

Therefore, all that has to be shown is that every element of $\Sigma_{1}$ in $L_{1}$ appears with every other element of $\Sigma_{1}$ in $L_{2}$. To prove this, recall that $B(x) \perp B(y)$. However, after removal of the $n_{2}$ transversals in $B(x)$ determined by the $n_{2}$ elements of $S$, and $n_{2}$ transversals in $B(y)$ determined by the $n_{2}$ elements of $T$, the following $2 n_{2} n_{1}$ pairs have been lost: 


$$
\left(x \alpha_{i}+\alpha_{j}, y \alpha_{i}+\alpha_{j}\right) \text { with } \alpha_{i}+\alpha_{j}=\gamma \in \Omega
$$

But the condition of the lemma guarantees the capture of these lost pairs by the $2 n_{2} n_{1}$ border cells.

The following example elucidates Lemma 5.1.

EXAMPLE 5.1. Let $n=10=7+3$ with

$$
\Sigma_{1}=\operatorname{GF}(7)=\{0,1,2,3,4,5,6\} \quad \text { and } \quad \Sigma_{2}=\{7,8,9\}
$$

Set $x=2, y=5$. Then

$$
B(2)=\begin{array}{llllllllllllll}
0 & 1 & 2 & 3 & 4 & 5 & 6 \\
2 & 3 & 4 & 5 & 6 & 0 & 1 \\
4 & 5 & 6 & 0 & 1 & 2 & 3 \\
6 & 0 & 1 & 2 & 3 & 4 & 5 \\
1 & 2 & 3 & 4 & 5 & 6 & 0 \\
3 & 4 & 5 & 6 & 0 & 1 & 2 \\
5 & 6 & 0 & 1 & 2 & 3 & 4
\end{array} \quad \begin{array}{llllllll}
0 & 5 & 6 & 0 & 1 & 2 & 3 & 4 \\
3 & 4 & 5 & 6 & 0 & 1 & 2 \\
1 & 2 & 3 & 4 & 5 & 6 & 0 \\
6 & 0 & 1 & 2 & 3 & 4 & 5 \\
4 & 5 & 6 & 0 & 1 & 2 & 3 \\
2 & 2 & 3 & 4 & 5 & 6 & 0 & 1
\end{array} .
$$

In order to locate the common parallel transversals in $B(x)$ and $B(y)$ the square $B(1)$ is exhibited below:

$$
B(1)=\begin{array}{lllllll}
0 & 1 & 2 & 3 & 4 & 5 & 6 \\
1 & 2 & 3 & 4 & 5 & 6 & 0 \\
2 & 3 & 4 & 5 & 6 & 0 & 1 \\
3 & 4 & 5 & 6 & 0 & 1 & 2 \\
4 & 5 & 6 & 0 & 1 & 2 & 3 \\
5 & 6 & 0 & 1 & 2 & 3 & 4 \\
6 & 0 & 1 & 2 & 3 & 4 & 5
\end{array}
$$

Let also

$$
A_{1}=\begin{array}{lll}
7 & 8 & 9 \\
8 & 9 & 7 \\
9 & 7 & 8
\end{array}, A_{2}=\begin{array}{lll}
7 & 8 & 9 \\
9 & 7 & 8 \\
8 & 9 & 7
\end{array} .
$$

Select $\Omega=\{0,1,2,3,4,5\}$ and $\Omega=S \cup T=\{0,1,3\} \cup\{2,4,5\}$. Now notice that for the following pairing of $s$ 's and $t$ 's 


$$
\begin{aligned}
k_{v}(x, y, s, t)=k_{v}(2,5, s, t) & =5 \text { for } s=0, t=4, \\
= & 4 \text { for } s=1, t=2, \\
= & 2 \text { for } s=3, t=5
\end{aligned}
$$

and

$$
\begin{array}{r}
k_{h}(x, y, s, t)=k_{h}(2,5, s, t)=1 \text { for } s=0, t=4 \\
=3 \text { for } s=1, t=2 \\
=0 \text { for } s=3, t=5 .
\end{array}
$$

Therefore for these pairings, $K_{v}=\{5,4,2\}$ and $K_{h}=\{1,3,0\}$, and thus $K_{v}$ $\cup K_{h}=\Omega$. Here $K_{v}=T$ and $K_{h}=S$. But in general there is no such requirement.

Now assembling all the parts $L_{1}$ and $L_{2}$ become:

$L_{1}=$\begin{tabular}{llllllll|lll|}
7 & 8 & 2 & 9 & 4 & 5 & 6 & 0 & 1 & 3 \\
8 & 3 & 9 & 5 & 6 & 0 & 7 & 1 & 2 & 4 \\
4 & 9 & 6 & 0 & 1 & 7 & 8 & 2 & 3 & 5 \\
9 & 0 & 1 & 2 & 7 & 8 & 5 & 3 & 4 & 6 \\
1 & 2 & 3 & 7 & 8 & 6 & 9 & 4 & 5 & 0 \\
3 & 4 & 7 & 8 & 0 & 9 & 2 & 5 & 6 & 1 \\
5 & 7 & 8 & 1 & 9 & 3 & 4 & 6 & 0 & 2 \\
\hline 0 & 6 & 5 & 4 & 3 & 2 & 1 & 7 & 8 & 9 \\
2 & 1 & 0 & 6 & 5 & 4 & 3 & 8 & 9 & 7 \\
6 & 5 & 4 & 3 & 2 & 1 & 0 & 9 & 7 & 8 \\
\hline
\end{tabular}

and $L_{2}\left(\begin{array}{cccccccc|ccc|}\hline 0 & 1 & 9 & 3 & 7 & 8 & 6 & 4 & 2 & 5 \\ 5 & 9 & 0 & 7 & 8 & 3 & 4 & 1 & 6 & 2 \\ 9 & 4 & 7 & 8 & 0 & 1 & 2 & 5 & 3 & 6 \\ 1 & 7 & 8 & 4 & 5 & 6 & 9 & 2 & 0 & 3 \\ 7 & 8 & 1 & 2 & 3 & 9 & 5 & 6 & 4 & 0 \\ 8 & 5 & 6 & 0 & 9 & 2 & 7 & 3 & 1 & 4 \\ 2 & 3 & 4 & 9 & 6 & 7 & 8 & 0 & 5 & 1 \\ \hline 6 & 2 & 5 & 1 & 4 & 0 & 3 & 7 & 8 & 9 \\ 3 & 6 & 2 & 5 & 1 & 4 & 0 & 9 & 7 & 8 \\ 4 & 0 & 3 & 6 & 2 & 5 & 1 & 8 & 9 & 7 \\ \hline\end{array}\right.$

The reader can satisfy his curiosity by direct checking that $L_{1}$ and $L_{2}$ is a pair of orthogonal Latin squares of order 10.

REMARK 5.2 The major problems with regard to the construction of an $\mathrm{O}(n, 2)$ via sum composition are the following:

(i) Choice of $x$ and $y$. It is found that $y=x^{-1}$ simplifies the calculations considerably.

(ii) Selection of the set $\Omega$ from the $\left(\begin{array}{c}n_{1} \\ 2 n_{2}\end{array}\right)$ possible choices.

(iii) Splitting of $\Omega$ into $S \cup T$.

(iv) Projection (vertically and horizontally) of the members of $S$ and $T$, in the formation of $L_{1}$ and $L_{2}$, if possible, so that $K_{v} \cup K_{h}=\Omega$. 
A backward solution of the problem, especially in the case $y=x^{-1}$, is easier, namely, devise any "admissible scheme" of capturing the members of $\Omega$ via vertical or horizontal projections. By an "admissible scheme" it is meant one should never let

$$
k_{v}(x, y, s, t)=s \text { or } t
$$

or

$$
k_{h}(x, y, s, t)=s \text { or } t
$$

since it is impossible to capture $s$ or $t$ through the pair $(s, t)$. Then the problem reduces to solving a system of $2 n_{2}$ homogenous equations in $2 n_{2}$ unknowns. The entries of the related matrix are in terms of $x$ and $y$. Now the question is: for what $x$ and $y$ and in what finite field does this system have a nontrivial solution with distinct components? Summing up the $2 n_{2}$ equations, $\left(\Sigma s_{i}-\Sigma t_{i}\right)(1-x y) /(x-1)=0$. This equation is independent of either the value of $n_{2}$ or the finite field in which the equations are supposed to hold. Thus the system of equations has no trivial solutions provided that either $x y=1$ or $\Sigma s_{i}=\Sigma t_{i}$. This justifies further the relation $x y=1$ used here to simplify the calculations. However $x y=1$ does not yet solve the problem because, in addition, the solution has to consist of distinct components. In cases investigated this leads to the reduction of the rank to $2 n_{2}-2$ and consequently to a condition that $y$ has to be a root of some polynomial. Whenever the polynomial was of degree two the finite fields in which the components of the solutions were distinct could be characterized easily. The difficulties arose when $y$ had to be a root of a polynomial of degree higher than two since there are no readily available tools to characterize such fields.

6. Construction of families of $0\left(n_{1}+n_{2}, 2\right)$ with the maximum value of $n_{2}$. As mentioned in $\$ 5$ the maximum value that $n_{2}$ can take is $\left[n_{1} / 2\right]$. A family of $0(n, 2), n=n_{1}+n_{2}$ where $n_{2}$ takes its maximum value is presented below.

THEOREM 6.1. For any prime $p$ and any positive integer $\alpha$ such that $n_{1}$ $=p^{\alpha} \geq 7, n_{1} \neq 13$, one can construct an $\mathrm{O}(n, 2)$ with the sum composition of an $\mathrm{O}\left(n_{1}, 2\right)$ based on $\mathrm{GF}\left(n_{1}\right)$ and any $\mathrm{O}\left(n_{2}, 2\right)$ where $n_{2}=\left[n_{1} / 2\right]$.

Proof. (By construction.) Let $\Sigma_{1}=\mathrm{GF}\left(n_{1}\right)$ and $\Sigma_{2}$ be any set of cardinality $n_{2}$ such that $\Sigma_{1} \cap \Sigma_{2}=\varnothing$. Let $\{B(x), B(y)\}$ be an $0\left(n_{1}, 2\right)$ based on $\mathrm{GF}\left(n_{1}\right)$ and $\left\{A_{1}, A_{2}\right\}$ any $0\left(n_{2}, 2\right)$ based on $\Sigma_{2}$. Let $\lambda \in \mathrm{GF}\left(n_{1}\right), \lambda \neq 0$ if $n_{1}$ is even. Let also $\Omega=\mathrm{GF}\left(n_{1}\right)-\{\lambda / 2\}=S \cup T$ such that for any $s \in$ 
$S$ there is $t \in T$ such that $s+t=\lambda$. Construct a Latin square $L_{1}$ by the sum composition of $B(x)$ and $A_{1}$ using any arbitrary vertical and horizontal projections of the $n_{2}$ parallel transversals determined by the elements of $S$. Now construct the Latin square $L_{2}$ by the sum composition of $B(y)$, and $A_{2}$ using the $n_{2}$ transversals in $B(y)$ determined by the elements of $T$ and the following projection rules: Project transversal $t_{i}$ on the row (column) which, upon superposition of $L_{2}$ on $L_{1}$, falls on the row (column) stemming from the transversal $s_{i}=\lambda-t_{i}$. Now by (4.7)

$$
k_{v}(y, s, t)+k_{h}(y, s, t)=(s+t)+(t-s)(x y-1) /(x-y),
$$

therefore if $x=y^{-1}$ then for $s_{1} \neq s_{2}$,

and

$$
k_{v}\left(y, s_{1}, \lambda-s_{1}\right) \neq k_{v}\left(y, s_{2}, \lambda-s_{2}\right)
$$

$$
k_{h}\left(y, s_{1}, \lambda-s_{1}\right) \neq k_{h}\left(y, s_{2}, \lambda-s_{2}\right)
$$

This implies that $K_{v} \cup K_{h}$ has cardinality $n_{1}-1$ and $K_{v} \cup K_{h}=\Omega$, and thus by Lemma 5.2 the set $\left\{L_{1}, L_{2}\right\}$ is an $\mathrm{O}(n, 2)$ on $\Sigma_{1} \cup \Sigma_{2}$.

REMARK 6.1. The method of Theorem 6.1 fails for $n_{1}=13$ only because there is no $\mathrm{O}(6,2)$. Otherwise, there will be no orthogonality contradiction on the other parts of $L_{1}$ and $L_{2}$ with their $6 \times 6$ lower right corner missing.

COROLLARY 6.1. The method of Theorem 6.1 produces infinitely many pairs of orthogonal Latin squares each of order $4 t+2$.

Proof. Let $p \equiv 7(\bmod 8)$ and $\alpha$ odd, then $p^{\alpha}=(8 t+5) / 3$ and thus $n_{1}$ $+n_{2}=4 t+2$.

COROLlaRY 6.2. If $p^{\alpha}>7$, then the composed orthogonal Latin squares in Theorem 6.1 have at least one common transversal if the corner $\mathrm{O}\left(n_{2}, 2\right)$ has a common transversal.

Proof. The original $\mathrm{O}\left(p^{\alpha}, 2\right)$ has $p^{\alpha}$ common parallel transversals. Therefore after removing $p^{\alpha}-1$ common parallel transversals there is still one common transversal in the corresponding portion of $\mathrm{O}\left(p^{\alpha}, 2\right)$ in the composed $\mathrm{O}(n, 2)$. This common transversal together with the assumed common transversal in the lower right corner $\mathrm{O}\left(n_{2}, 2\right)$ form a common transversal for the composed $\mathrm{O}(n, 2)$. The reason for the exclusion of $p^{\alpha}=$ 7 is the fact that no $\mathrm{O}(3,2)$ with a common transversal exists (see Lemma 2.2). 
REMARK 6.2 $\mathrm{O}(n, 2)$ with common parallel transversals have an application for the construction of a family of designs for two successive experiments (see Hedayat, Parker and Federer [7]).

The method of Theorem 6.1 will be clarified now by two examples, one for $n_{1}$ odd and one for $n_{1}$ even.

EXAMPLE 6.1. Let $n_{1}=7, \operatorname{GF}(7)=\{0,1, \ldots, 6\}$. Then for $y=3, x=$ $y^{-1}=5$ we have $\{B(1), B(5), B(3)\}=$

$\begin{array}{lllllllllllllllllllll}0 & 1 & 2 & 3 & 4 & 5 & 6 & 0 & 1 & 2 & 3 & 4 & 5 & 6 & 0 & 1 & 2 & 3 & 4 & 5 & 6\end{array}$

$\begin{array}{lllllllllllllllllllll}1 & 2 & 3 & 4 & 5 & 6 & 0 & 5 & 6 & 0 & 1 & 2 & 3 & 4 & 3 & 4 & 5 & 6 & 0 & 1 & 2\end{array}$

$\begin{array}{lllllllllllllllllllll}2 & 3 & 4 & 5 & 6 & 0 & 1 & 3 & 4 & 5 & 6 & 0 & 1 & 2 & 6 & 0 & 1 & 2 & 3 & 4 & 5\end{array}$

$\begin{array}{llllllllllllllllllllll}3 & 4 & 5 & 6 & 0 & 1 & 2 & 1 & 2 & 3 & 4 & 5 & 6 & 0 & 2 & 3 & 4 & 5 & 6 & 0 & 1\end{array}$.

$\begin{array}{llllllllllllllllllllll}4 & 5 & 6 & 0 & 1 & 2 & 3 & 6 & 0 & 1 & 2 & 3 & 4 & 5 & 5 & 6 & 0 & 1 & 2 & 3 & 4\end{array}$

$\begin{array}{lllllllllllllllllllll}5 & 6 & 0 & 1 & 2 & 3 & 4 & 4 & 5 & 6 & 0 & 1 & 2 & 3 & 1 & 2 & 3 & 4 & 5 & 6 & 0\end{array}$

$\begin{array}{lllllllllllllllllllll}6 & 0 & 1 & 2 & 3 & 4 & 5 & 2 & 3 & 4 & 5 & 6 & 0 & 1 & 4 & 5 & 6 & 0 & 1 & 2 & 3\end{array}$

For $n_{2}=\left(n_{1}-1\right) / 2$, let $\Sigma_{2}=\{7,8,9\}$ and

$$
\left\{A_{1}, A_{2}\right\}=\begin{array}{llllll}
7 & 8 & 9 & 7 & 8 & 9 \\
8 & 9 & 7 & 9 & 7 & 8 \\
9 & 7 & 8 & 8 & 9 & 7
\end{array} .
$$

Finally for $\lambda=1, S=\{1,2,3\}$ and $T=\{0,6,5\}$ we have $\left\{L_{1}, L_{2}\right\}=$

\begin{tabular}{lllllll|lll}
0 & 7 & 8 & 9 & 4 & 5 & 6 & 1 & 2 & 3 \\
7 & 8 & 9 & 1 & 2 & 3 & 4 & 5 & 6 & 0 \\
8 & 9 & 5 & 6 & 0 & 1 & 7 & 2 & 3 & 4 \\
9 & 2 & 3 & 4 & 5 & 7 & 8 & 6 & 0 & 1 \\
6 & 0 & 1 & 2 & 7 & 8 & 9 & 3 & 4 & 5 \\
4 & 5 & 6 & 7 & 8 & 9 & 3 & 0 & 1 & 2 \\
2 & 3 & 7 & 8 & 9 & 0 & 1 & 4 & 5 & 6 \\
\hline 5 & 1 & 4 & 0 & 3 & 6 & 2 & 7 & 8 & 9 \\
3 & 6 & 2 & 5 & 1 & 4 & 0 & 8 & 9 & 7 \\
1 & 4 & 0 & 3 & 6 & 2 & 5 & 9 & 7 & 8
\end{tabular}

\begin{tabular}{lllllll|lll}
7 & 1 & 2 & 3 & 4 & 9 & 8 & 0 & 6 & 5 \\
3 & 4 & 5 & 6 & 9 & 8 & 7 & 2 & 1 & 0 \\
6 & 0 & 1 & 9 & 8 & 7 & 5 & 4 & 3 & 2 \\
2 & 3 & 9 & 8 & 7 & 0 & 1 & 6 & 5 & 4 \\
5 & 9 & 8 & 7 & 2 & 3 & 4 & 1 & 0 & 6 \\
9 & 8 & 7 & 4 & 5 & 6 & 0 & 3 & 2 & 1 \\
8 & 7 & 6 & 0 & 1 & 2 & 9 & 5 & 4 & 3 \\
\hline 0 & 5 & 3 & 1 & 6 & 4 & 2 & 7 & 8 & 9 \\
4 & 2 & 0 & 5 & 3 & 1 & 6 & 9 & 7 & 8 \\
1 & 6 & 4 & 2 & 0 & 5 & 3 & 8 & 9 & 7
\end{tabular}

which is an $0(10,2)$. 
EXAMPLE 6.2. Let $n_{1}=8, \mathrm{GF}(8)=\{0,1, \ldots, 7\}$ with the following addition $(+)$ and multiplication $(X)$ tables:

\begin{tabular}{|l|llllllll|}
\hline+ & 0 & 1 & 2 & 3 & 4 & 5 & 6 & 7 \\
\hline 0 & 0 & 1 & 2 & 3 & 4 & 5 & 6 & 7 \\
1 & 1 & 0 & 6 & 4 & 3 & 7 & 2 & 5 \\
2 & 2 & 6 & 0 & 7 & 5 & 4 & 1 & 3 \\
3 & 3 & 4 & 7 & 0 & 1 & 6 & 5 & 2 \\
4 & 4 & 3 & 5 & 1 & 0 & 2 & 7 & 6 \\
5 & 5 & 7 & 4 & 6 & 2 & 0 & 3 & 1 \\
6 & 6 & 2 & 1 & 5 & 7 & 3 & 0 & 4 \\
7 & 7 & 5 & 3 & 2 & 6 & 1 & 4 & 0 \\
\hline
\end{tabular}

\begin{tabular}{|c|llllllll|}
\hline$\times$ & 0 & 1 & 2 & 3 & 4 & 5 & 6 & 7 \\
\hline 0 & 0 & 0 & 0 & 0 & 0 & 0 & 0 & 0 \\
1 & 0 & 1 & 2 & 3 & 4 & 5 & 6 & 7 \\
2 & 0 & 2 & 3 & 4 & 5 & 6 & 7 & 1 \\
3 & 0 & 3 & 4 & 5 & 6 & 7 & 1 & 2 \\
4 & 0 & 4 & 5 & 6 & 7 & 1 & 2 & 3 \\
5 & 0 & 5 & 6 & 7 & 1 & 2 & 3 & 4 \\
6 & 0 & 6 & 7 & 1 & 2 & 3 & 4 & 5 \\
7 & 0 & 7 & 1 & 2 & 3 & 4 & 5 & 6 \\
\hline
\end{tabular}

Then for $y=3, x=y^{-1}=6,\{B(1), B(6), B(3)\}=$

$\begin{array}{llllllllllllllllllllllll}0 & 1 & 2 & 3 & 4 & 5 & 6 & 7 & 0 & 1 & 2 & 3 & 4 & 5 & 6 & 7 & 0 & 1 & 2 & 3 & 4 & 5 & 6 & 7\end{array}$ $\begin{array}{llllllllllllllllllllllll}1 & 0 & 6 & 4 & 3 & 7 & 2 & 5 & 6 & 2 & 1 & 5 & 7 & 3 & 0 & 4 & 3 & 4 & 7 & 0 & 1 & 6 & 5 & 2\end{array}$ $\begin{array}{llllllllllllllllllllllll}2 & 6 & 0 & 7 & 5 & 4 & 1 & 3 & 7 & 5 & 3 & 2 & 6 & 1 & 4 & 0 & 4 & 3 & 5 & 1 & 0 & 2 & 7 & 6\end{array}$

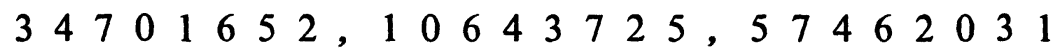
$\begin{array}{llllllllllllllllllllllll}4 & 3 & 5 & 1 & 0 & 2 & 7 & 6 & 2 & 6 & 0 & 7 & 5 & 4 & 1 & 3 & 6 & 2 & 1 & 5 & 7 & 3 & 0 & 4\end{array}$ $\begin{array}{llllllllllllllllllllllll}5 & 7 & 4 & 6 & 2 & 0 & 3 & 1 & 3 & 4 & 7 & 0 & 1 & 6 & 5 & 2 & 7 & 5 & 3 & 2 & 6 & 1 & 4 & 0\end{array}$ $\begin{array}{llllllllllllllllllllllll}6 & 2 & 1 & 5 & 7 & 3 & 0 & 4 & 4 & 3 & 5 & 1 & 0 & 2 & 7 & 6 & 1 & 0 & 6 & 4 & 3 & 7 & 2 & 5\end{array}$ $\begin{array}{llllllllllllllllllllllll}75 & 3 & 2 & 6 & 1 & 4 & 0 & 5 & 7 & 4 & 6 & 2 & 0 & 3 & 1 & 2 & 6 & 0 & 7 & 5 & 4 & 1 & 3\end{array}$

For $n_{2}=n_{1} / 2=4$, let $\Sigma_{2}=\{A, B, C, D\}$ and

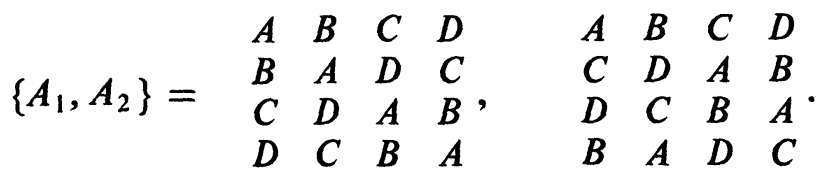


Finally for $\lambda=2, S=\{0,1,3,4\}$, and $T=\{2,6,7,5\}$ one obtains $\left\{L_{1}, L_{2}\right\}=$

\begin{tabular}{llllllll|llll}
$A$ & $B$ & 2 & $C$ & $D$ & 5 & 6 & 7 & 0 & 1 & 3 & 4 \\
$B$ & $A$ & 1 & $D$ & $C$ & 3 & 0 & 4 & 2 & 6 & 7 & 5 \\
7 & 5 & $A$ & 2 & 6 & $D$ & $B$ & $C$ & 3 & 4 & 0 & 1 \\
$C$ & $D$ & 6 & $A$ & $B$ & 7 & 2 & 5 & 4 & 3 & 1 & 0 \\
$D$ & $C$ & 0 & $B$ & $A$ & 4 & 1 & 3 & 5 & 7 & 6 & 2 \\
3 & 4 & $D$ & 0 & 1 & $A$ & $C$ & $B$ & 6 & 2 & 5 & 7 \\
4 & 3 & $B$ & 1 & 0 & $C$ & $A$ & $D$ & 7 & 5 & 2 & 6 \\
5 & 7 & $C$ & 6 & 2 & $B$ & $D$ & $A$ & 1 & 0 & 4 & 3 \\
\hline 0 & 2 & 3 & 4 & 5 & 6 & 7 & 1 & $A$ & $B$ & $C$ & $D$ \\
6 & 1 & 5 & 7 & 3 & 0 & 4 & 2 & $B$ & $A$ & $D$ & $C$ \\
1 & 6 & 4 & 3 & 7 & 2 & 5 & 0 & $C$ & $D$ & $A$ & $B$ \\
2 & 0 & 7 & 5 & 4 & 1 & 3 & 6 & $D$ & $C$ & $B$ & $A$
\end{tabular}

\begin{tabular}{llllllll|llll}
0 & 1 & $A$ & 3 & 4 & $D$ & $B$ & $C$ & 2 & 6 & 7 & 5 \\
3 & 4 & $B$ & 0 & 1 & $C$ & $A$ & $D$ & 5 & 7 & 6 & 2 \\
$A$ & $B$ & 5 & $C$ & $D$ & 2 & 7 & 6 & 4 & 3 & 1 & 0 \\
5 & 7 & $C$ & 6 & 2 & $B$ & $D$ & $A$ & 1 & 0 & 4 & 3 \\
6 & 2 & $D$ & 5 & 7 & $A$ & $C$ & $B$ & 3 & 4 & 0 & 1 \\
$D$ & $C$ & 3 & $B$ & $A$ & 1 & 4 & 0 & 6 & 2 & 5 & 7 \\
$B$ & $A$ & 6 & $D$ & $C$ & 7 & 2 & 5 & 0 & 1 & 3 & 4 \\
$C$ & $D$ & 0 & $A$ & $B$ & 4 & 1 & 3 & 7 & 5 & 2 & 6 \\
\hline 4 & 0 & 2 & 7 & 6 & 3 & 5 & 1 & $A$ & $B$ & $C$ & $D$ \\
1 & 3 & 7 & 2 & 5 & 0 & 6 & 4 & $C$ & $D$ & $A$ & $B$ \\
2 & 5 & 4 & 1 & 3 & 6 & 0 & 7 & $D$ & $C$ & $B$ & $A$ \\
7 & 6 & 1 & 4 & 0 & 5 & 3 & 2 & $B$ & $A$ & $D$ & $C$
\end{tabular}

which is an $0(12,2)$.

7. Construction of families of $0\left(n_{1}+n_{2}, 2\right)$ with the minimum value of $\boldsymbol{n}_{2}$. Presently the problem of the construction of a set $\mathrm{O}(n, 2)$ for $n=n_{1}+3$, $n_{1}=p^{\alpha}, p$ a prime greater than or equal to seven and $\alpha$ a positive integer will be investigated. It is clearly sufficient to show that the construction can be achieved for any $p \geq 7$. The proof can then be carried over to any $n_{1}=$ $p^{\alpha}$.

As before let $B(1), B(x)$, and $B(y)$ be three orthogonal Latin squares with elements in $\operatorname{GF}\left(n_{1}\right)$.

Let $S=\left\{s_{1}, s_{2}, s_{3}\right\}, T=\left\{t_{1}, t_{2}, t_{3}\right\}$ denote sets of transversals projected from $B(x)$ and $B(y)$ respectively.

The problem faced now is, can one choose the sets $S$ and $T$ in such a manner that the ranges of the two functions $k_{v}\left(x, y, s_{i}, t_{j}\right)$ and $k_{h}\left(x, y, s_{i}, t_{j}\right)$ for $i, j=1,2,3$ exhaust the sets $S \cup T$, and if so in what way, if any, does the choice depend on $x$ and $y$ ? This leads to the problem, how many distinct systems of choices are possible? Reducing the problem to nonisomorphic cases two cases are considered "distinct" if they cannot be obtained from each other by interchanging the squares, transposing both squares, or permuting the elements within each of the sets $S$ or $T$. Thus it may be assumed that $i=j$ for one of the functions, say $k_{v}\left(x, y, s_{i}, t_{j}\right)$, since this can be achieved by permuting the elements of one of the sets $S$ or $T$. Furthermore it is assumed that the range of the function $k_{v}\left(x, y, s_{i}, t_{j}\right)$ consists of either two or three elements of the set $S$. Cases in which the range includes none or one element of $S$ can be obtained from the above by interchang- 
ing the sets $S$ and $T$ and the functions $k_{\nu}$ and $k_{h}$. To facilitate the notation the arguments $x$ and $y$ will be omitted in the present considerations.

In view of the above, there are just four distinct patterns for the range of $k_{v}(s, t)$. They are:

$\begin{array}{cccc}\text { I } & \text { II } & \text { III } & \text { IV } \\ k_{v}\left(s_{1}, t_{1}\right)=s_{2} & k_{v}\left(s_{1}, t_{1}\right)=s_{2} & k_{v}\left(s_{1}, t_{1}\right)=s_{2} & k_{v}\left(s_{1}, t_{1}\right)=s_{2} \\ k_{v}\left(s_{2}, t_{2}\right)=s_{3} & k_{v}\left(s_{2}, t_{2}\right)=s_{3} & k_{v}\left(s_{2}, t_{2}\right)=s_{3} & k_{v}\left(s_{2}, t_{2}\right)=s_{1} \\ k_{v}\left(s_{3}, t_{3}\right)=s_{1} & k_{v}\left(s_{3}, t_{3}\right)=t_{1} & k_{v}\left(s_{3}, t_{3}\right)=t_{2} & k_{v}\left(s_{3}, t_{3}\right)=t_{1}\end{array}$

For each of these patterns there are twelve distinct possibilities for the range of $k_{h}\left(s_{i}, t_{j}\right)$. Thus there are a total of 48 cases to be considered.

In [8] it was assumed that $x y=1$. This seemed to simplify the calculations. Ruiz and Seiden [9] showed that a necessary and sufficient condition for obtaining nontrivial solutions for the systems of equations arising in the method of sum composition is either $\Sigma s_{i}=\Sigma t_{i}$ or $x y=1$. They also showed that for patterns II, III and IV the elements of $S \cup T$ cannot be distinct unless $x y=1$.

It is shown here that under the assumption $x y=1$ one cannot construct a set $0\left(n_{1}+3,2\right)$ for some primes, $n_{1}$, of the form $60 m+11$ or $60 m+$ 59. However using pattern $I$ and another relation between $x$ and $y$ this gap can be bridged.

All 48 distinct systems of equations will be investigated below under the assumption $x y=1$. This assumption, as mentioned before, reduces the rank of the system to at most five. However imposing the additional condition that the solutions must be distinct reduces the rank in all cases to at most four and yields a condition that $y$ must be a root of certain equations. There are cases in which $y$ has to satisfy either the equation $y=$ 0 or $y=1$. Clearly this is incompatible with the condition $x y=1, x \neq y$. In other cases the problem reduces to solving either a quadratic or a fourth degree equation in $y$ in a Galois field. The cases of quadratic equations, however, can be easily analyzed. The latter helps in establishing that if $x y$ $=1$, there are primes for which a set $\mathrm{O}\left(n_{1}+3,2\right)$ cannot be constructed.

The cases in which $y$ has to be a root of a quadratic equation separate the primes for which a set $\mathrm{O}\left(n_{1}+3,2\right)$ can be constructed into four classes, not necessarily disjoint. These are such that either $-1,-2,-3$, or -15 are quadratic residues in $\mathrm{GF}\left(n_{1}\right)$. A representative pattern for each of these classes is presented. 
Case 1.

$$
\begin{array}{ll}
k_{v}\left(s_{1}, t_{1}\right)=s_{2} & k_{h}\left(s_{1}, t_{2}\right)=t_{1} \\
k_{v}\left(s_{2}, t_{2}\right)=s_{3} & k_{h}\left(s_{2}, t_{1}\right)=t_{3} \\
k_{v}\left(s_{3}, t_{3}\right)=s_{1} & k_{h}\left(s_{3}, t_{3}\right)=t_{2} .
\end{array}
$$

This system will be of rank four and will exhaust the elements of the set $S$ $\cup T$ provided that $y$ is a root of the equation $2 y^{2}+3 y+3=0$. Hence $y=$ $(-3 \pm \sqrt{-15}) / 4$, and the system will have solutions provided that either -3 and 5 or -5 and 3 are quadratic residues $\bmod p$.

A system of solutions is:

$$
\begin{aligned}
& s_{1}=\left(y^{2}+y+1\right) s_{2}-y(y+1) t_{2} \\
& t_{1}=-y s_{2}+(y+1) t_{2} \\
& s_{3}=s_{2} /(y+1)+y t_{2} /(y+1) \\
& t_{3}=\left(y^{3}+y^{2}+y\right) s_{2} /(y+1)-\left(y^{3}+y^{2}-1\right) /(y+1) .
\end{aligned}
$$

Case 2.

$$
\begin{array}{ll}
k_{v}\left(s_{1}, t_{1}\right)=s_{2} & k_{h}\left(s_{1}, t_{2}\right)=t_{3} \\
k_{v}\left(s_{2}, t_{2}\right)=s_{3} & k_{h}\left(s_{2}, t_{1}\right)=s_{1} \\
k_{v}\left(s_{3}, t_{3}\right)=t_{1} & k_{h}\left(s_{3}, t_{3}\right)=t_{2}
\end{array}
$$

For this system to be solvable and exhaust the set $S \cup T, y$ has to be a root of the equation $y^{2}+y+1=0$, i.e., -3 has to be quadratic residue, i.e. $p$ has to be of the form $6 m+1$. The following forms a system of solutions:

$$
\begin{aligned}
& s_{1}=-s_{3} /(y+1)+(y+2) t_{3} /(y+1) \\
& s_{2}=(2 y+1) s_{3} /(y+1)-y t_{3} /(y+1) \\
& t_{1}=s_{3} /(y+1)+y t_{3} /(y+1) \\
& t_{2}=y s_{3} /(y+1)+t_{3} /(y+1) .
\end{aligned}
$$

Case 3.

$$
\begin{array}{ll}
k_{v}\left(s_{1}, t_{1}\right)=s_{2} & k_{h}\left(s_{1}, t_{1}\right)=t_{3} \\
k_{v}\left(s_{2}, t_{2}\right)=s_{3} & k_{h}\left(s_{2}, t_{2}\right)=s_{1} \\
k_{v}\left(s_{3}, t_{3}\right)=t_{1} & k_{h}\left(s_{3}, t_{3}\right)=t_{2} .
\end{array}
$$

This system will be of rank four and the solutions will exhaust the set $S \cup T$ provided that $y$ is a root of the equation $2 y^{2}+1=0$, i.e., -2 is a quadratic residue of $p=8 m+1$ or $8 m+3$.

A system of solutions is: 


$$
\begin{aligned}
& s_{3}=y s_{1}+(1-y) s_{2} \\
& t_{1}=2 y s_{1}-(2 y-1) s_{2} \\
& t_{2}=(1+y) s_{1}-y s_{2} \\
& t_{3}=(1+2 y) s_{1}-2 y s_{2} .
\end{aligned}
$$

Case 4.

$$
\begin{array}{ll}
k_{v}\left(s_{1}, t_{1}\right)=s_{2} & k_{h}\left(s_{1}, t_{1}\right)=t_{3} \\
k_{v}\left(s_{2}, t_{2}\right)=s_{3} & k_{h}\left(s_{2}, t_{3}\right)=s_{1} \\
k_{v}\left(s_{3}, t_{3}\right)=t_{2} & k_{h}\left(s_{3}, t_{2}\right)=t_{1} .
\end{array}
$$

This system will be of rank four and exhaust the set $S \cup T$ provided that $y^{2}$ $+1=0$, i.e., -1 has to be a quadratic residue or $p=4 m+1$.

A system of solutions is:

$$
\begin{aligned}
& s_{2}=s_{1} /(y+1)+y t_{1} /(y+1) \\
& t_{2}=-s_{1} /(y+1)-\left(y^{2}-y-1\right) t_{1} /(y+1) \\
& s_{3}=s_{1} / y(y+1)+(2 y+1) t_{1} /(y+1) \\
& t_{3}=y s_{1} /(y+1)+y t_{1} /(y+1) .
\end{aligned}
$$

Since case 4 captures all primes of the form $4 m+1$ the problem is: Are there primes of the form $4 m+3$ which are not captured by the remaining three cases? Case 3 captures all primes of the form $4 m+3$ for $m$ even. Case 2 captures primes of the form $4 m+3$ for $m$ odd, provided that they are also of the form $6 m+1$. Hence cases 2, 3 and 4 omit primes of the form $12 m+$ 11 for $m$ odd. Case 1 captures two families of these primes, provided that they are also of the form $60 m+23=12(5 m+1)+11$ or $60 m+47=$ $12(5 m+3)+11$. Thus none of the four cases capture primes of the form $12(5 m+2)+11$ or $12(5 m+4)+11$.

The next question asked is whether the failure to capture the above mentioned primes is due to the restriction $x y=1$ ? Could one, assuming $x y$ $\neq 1$ but $\Sigma s_{i}=\Sigma t_{i}$, supplement the missing primes? The answer is in the affirmative.

It is shown that keeping the assumption $x y=1$, one may capture some but not all of the missing primes. As mentioned before some of the 48 cases lead to conditions that $y$ has to satisfy a fourth degree equation. There are five equations of degree four as follows:

$$
\begin{aligned}
& \text { 1. } y^{4}+2 y^{3}+3 y^{2}+y+1=0 \\
& \text { 2. } y^{4}+2 y^{3}+3 y^{2}+3 y+2=0 \\
& \text { 3. } y^{4}+3 y^{3}+6 y^{2}+5 y+2=0
\end{aligned}
$$




$$
\begin{aligned}
& y^{4}+2 y^{3}+4 y^{2}+4 y+2=0 \\
& \text { 5. } y^{4}+3 y^{3}+6 y^{2}+6 y+3=0
\end{aligned}
$$

Equations 4 and 5 cannot have a linear factor unless $p$ is of the form $6 m+$ 1 or $4 m+1$ respectively. Hence these patterns cannot yield primes not obtainable otherwise. It can also be seen that the set of primes which can be captured by equations 1 and 2 are identical. Hence the problem reduces to investigating one of the first two equations and equation 3 . Using the high speed computer facilities at Michigan State University it was found that some but not all of the missing primes of the form $60 m+11$ and $60 m+59$ can be captured by these equations.

The first 10 primes of the two missing types of primes were investigated. It was found that in $\mathrm{GF}(191)$ and $\mathrm{GF}(1319)$ both equations did not have a linear factor and each of these primes is the smallest in its class.

It may be worthwhile mentioning that the number of solutions in the cases investigated was the same for both equations. It is suspected that this holds for all finite fields but we lack the tools to investigate the problem. We would like to have a method to characterize finite fields in which equations of degree greater than two have roots. This would prove very useful for our further research on sum composition.

Failing to construct a set of $\mathrm{O}\left(n_{1}+3,2\right)$ for $n_{1}$ of the form $60 m+11$ and $60 m+59$ with the assumption $x y=1$ it is natural to try to achieve, if possible, this goal with the alternative $\Sigma s_{i}=\Sigma t_{i}$. It will be shown that the choice $(1-x) /(x-y)=1$ will prove sufficient to capture the missing primes. Writing now case 1 in terms of $x$ produces:

$$
\begin{array}{ll}
s_{1}=2 x s_{3}-(2 x-1) t_{3} & t_{1}=2 s_{1}-t_{2} \\
s_{2}=2 x s_{1}-(2 x-1) t_{2} & t_{3}=2 s_{2}-t_{1} \\
s_{3}=2 x s_{2}-(2 x-1) t_{2} & t_{2}=2 s_{3}-t_{3} .
\end{array}
$$

This system will have rank four and yield distinct solutions for the unknowns provided that $x$ satisfies the equation $4 x^{2}-6 x+1=0$. Hence $x=$ $(3 \pm \sqrt{5}) / 4$. Thus this system will capture all primes $n_{1}$ such that 5 is a quadratic residue in $\operatorname{GF}\left(n_{1}\right)$, i.e., $n_{1}$ is of the form $5 m+1$ or $5 m+4$. Primes of the form $5 m+1$ and $5 m+4$ include primes of the form $60 m+$ 11 and $60 m+59$ respectively.

A system of solutions is:

$$
\begin{aligned}
& s_{1}=2 x s_{3}-(2 x-1) t_{3} \\
& t_{2}=2 s_{3}-t_{3} \\
& t_{1}=2(2 x-1) s_{3}-(4 x-3) t_{3} \\
& s_{2}=(2 x-1) s_{3}-2(x-1) t_{3} .
\end{aligned}
$$


The result of the previous discussion can be summarized in the following theorem.

THEOREM 7.1. Using the method of sum composition it is possible to construct a set $\mathrm{O}\left(p^{\alpha}+3,2\right)$ for all $p \geq 7$.

It is emphasized that the method of construction depends on the form of $p$ but not on its specific value.

COROLlaRY 7.1. The method of Theorem 7.1 produces an infinite family of $\mathrm{O}(n, 2)$ with $n=4 t+2$.

EXAMPLE 7.1. The pattern of case 3 can be applied to $O(11,2)$ to yield a set $\mathrm{O}(14,2)$. It will result in $x=3, y=4$ and $S=\{0,1,8\}, T=\{4,7,3\}$

\begin{tabular}{rrrrrrrrrrr|rrr}
$A$ & $B$ & 2 & 3 & 4 & 5 & 6 & 7 & $C$ & 9 & 10 & 0 & 1 & 8 \\
$B$ & 4 & 5 & 6 & 7 & 8 & 9 & $C$ & 0 & 1 & $A$ & 2 & 3 & 10 \\
6 & 7 & 8 & 9 & 10 & 0 & $C$ & 2 & 3 & $A$ & $B$ & 4 & 5 & 1 \\
9 & 10 & 0 & 1 & 2 & $C$ & 4 & 5 & $A$ & $B$ & 8 & 6 & 7 & 3 \\
1 & 2 & 3 & 4 & $C$ & 6 & 7 & $A$ & $B$ & 10 & 0 & 8 & 9 & 5 \\
4 & 5 & 6 & $C$ & 8 & 9 & $A$ & $B$ & 1 & 2 & 3 & 10 & 0 & 7 \\
7 & 8 & $C$ & 10 & 0 & $A$ & $B$ & 3 & 4 & 5 & 6 & 1 & 2 & 9 \\
10 & $C$ & 1 & 2 & $A$ & $B$ & 5 & 6 & 7 & 8 & 9 & 3 & 4 & 0 \\
$C$ & 3 & 4 & $A$ & $B$ & 7 & 8 & 9 & 10 & 0 & 1 & 5 & 6 & 2 \\
5 & 6 & $A$ & $B$ & 9 & 10 & 0 & 1 & 2 & 3 & $C$ & 7 & 8 & 4 \\
8 & $A$ & $B$ & 0 & 1 & 2 & 3 & 4 & 5 & $C$ & 7 & 9 & 10 & 6 \\
\hline 0 & 9 & 7 & 5 & 3 & 1 & 10 & 8 & 6 & 4 & 2 & $A$ & $B$ & $C$ \\
3 & 1 & 10 & 8 & 6 & 4 & 2 & 0 & 9 & 7 & 5 & $B$ & $C$ & $A$ \\
2 & 0 & 9 & 7 & 5 & 3 & 1 & 10 & 8 & 6 & 4 & $C$ & $A$ & $B$ \\
& & & & & & & & & & & & & \\
0 & 1 & 2 & $A$ & $B$ & 5 & 6 & $C$ & 8 & 9 & 10 & 4 & 7 & 3 \\
4 & 5 & $A$ & $B$ & 8 & 9 & $C$ & 0 & 1 & 2 & 3 & 7 & 10 & 6 \\
8 & $A$ & $B$ & 0 & 1 & $C$ & 3 & 4 & 5 & 6 & 7 & 10 & 2 & 9 \\
$A$ & $B$ & 3 & 4 & $C$ & 6 & 7 & 8 & 9 & 10 & 0 & 2 & 5 & 1 \\
$B$ & 6 & 7 & $C$ & 9 & 10 & 0 & 1 & 2 & 3 & $A$ & 5 & 8 & 4 \\
9 & 10 & $C$ & 1 & 2 & 3 & 4 & 5 & 6 & $A$ & $B$ & 8 & 0 & 7 \\
2 & $C$ & 4 & 5 & 6 & 7 & 8 & 9 & $A$ & $B$ & 1 & 0 & 3 & 10 \\
$C$ & 7 & 8 & 9 & 10 & 0 & 1 & $A$ & $B$ & 4 & 5 & 3 & 6 & 2 \\
10 & 0 & 1 & 2 & 3 & 4 & $A$ & $B$ & 7 & 8 & $C$ & 6 & 9 & 5 \\
3 & 4 & 5 & 6 & 7 & $A$ & $B$ & 10 & 0 & $C$ & 2 & 9 & 1 & 8 \\
7 & 8 & 9 & 10 & $A$ & $B$ & 2 & 3 & $C$ & 5 & 6 & 1 & 4 & 0 \\
\hline 5 & 2 & 10 & 7 & 4 & 1 & 9 & 6 & 3 & 0 & 8 & $A$ & $B$ & $C$ \\
6 & 3 & 0 & 8 & 5 & 2 & 10 & 7 & 4 & 1 & 9 & $C$ & $A$ & $B$ \\
1 & 9 & 6 & 3 & 0 & 8 & 5 & 2 & 10 & 7 & 4 & $B$ & $C$ & $A$
\end{tabular}


REMARK 7.1. For $n \leq 100$ of the form $4 t+2$ there are three instances where one can utilize either Theorem 6.1 or 7.1 to produce an $\mathrm{O}(n, 2)$. These orders are 34,46 and 70 which can be decomposed as follows:

$$
\begin{aligned}
& 34=23+11 \text { or } 31+3 \\
& 46=31+15 \text { or } 41+3 \\
& 70=47+23 \text { or } 67+3
\end{aligned}
$$

The natural question to ask now is in what direction should we extend the research on construction of orthogonal Latin squares using the method of sum composition? One obvious direction would be to investigate the problem of construction of a set $0(n, t)$ for $t>2$. As a first step in this direction it is necessary to extend the investigations beyond the extreme values of $n_{2}$ (see $\left.\S 10\right)$. The next two smallest values are $n_{2}=4,5$. As will be seen, the composed orthogonal Latin squares for these values of $n_{2}$ have a useful statistical application. These cases are considered in the following two sections.

8. Construction of two families of $\mathrm{O}\left(n_{1}+4,2\right)$. It is clear that an exhaustive search for patterns, as was done for $n_{2}=3$, would be very tedious. Preliminary investigations indicate that one could find patterns which would yield a set $0\left(n_{1}+4,2\right)$ for any $n_{1}=p^{\alpha}$ as long as $p \geq 11$ and $\alpha$ $\geq 1$. Here two families of $0\left(p^{\alpha}+4,2\right)$ for which either -1 or -2 are quadratic residues in $\operatorname{GF}\left(p^{\alpha}\right)$ will be presented. For both families one has to assume $x y=1$ in order to obtain distinct solutions for the unknowns in question.

Case 1.

$$
\begin{array}{ll}
k_{v}\left(s_{1}, t_{1}\right)=t_{3} & k_{h}\left(s_{1}, t_{4}\right)=t_{2} \\
k_{v}\left(s_{2}, t_{2}\right)=s_{1} & k_{h}\left(s_{2}, t_{3}\right)=t_{4} \\
k_{v}\left(s_{3}, t_{3}\right)=t_{1} & k_{h}\left(s_{3}, t_{2}\right)=s_{4} \\
k_{v}\left(s_{4}, t_{4}\right)=s_{2} & k_{h}\left(s_{4}, t_{1}\right)=s_{3} .
\end{array}
$$

This system of equations will have distinct solutions provided that $3 y^{2}+2 y$ $+1=0$, i.e., -2 has to be quadratic residue or $p$ has to be of the form $8 m$ +1 or $8 m+3$. A system of solutions in terms of $t_{1}$ and $t_{3}$ is:

$$
\begin{array}{ll}
s_{1}=y t_{1}+(y+1) t_{3} & s_{4}=(y+2) t_{1}-(y+1) t_{3} \\
s_{2}=-(y-1) t_{1}+y t_{3} & t_{2}=2(y+1) t_{1}-(2 y+1) t_{3} \\
s_{3}=(y+1) t_{1}-y t_{3} & t_{4}=(2 y+1) t_{1}-2 y t_{3} .
\end{array}
$$


Case 2.

$$
\begin{array}{ll}
k_{v}\left(s_{1}, t_{1}\right)=t_{2} & k_{h}\left(s_{1}, t_{1}\right)=s_{2} \\
k_{v}\left(s_{2}, t_{2}\right)=s_{1} & k_{h}\left(s_{2}, t_{2}\right)=t_{1} \\
k_{v}\left(s_{3}, t_{3}\right)=t_{4} & k_{h}\left(s_{3}, t_{3}\right)=s_{4} \\
k_{v}\left(s_{4}, t_{4}\right)=s_{3} & k_{h}\left(s_{4}, t_{4}\right)=t_{3} .
\end{array}
$$

Notice that the four equations of either lines one and two or three and four form a loop. These loops will yield distinct solutions provided that $y^{2}+1$ $=0$ or $p=4 m+1$. A system of solutions is:

$$
\begin{array}{ll}
t_{2}=\left(s_{1}+y t_{1}\right) /(y+1) & t_{4}=\left(s_{3}+y t_{3}\right) /(y+1) \\
s_{2}=\left(t_{1}+y s_{1}\right) /(y+1) & s_{4}=\left(t_{3}+y s_{3}\right) /(y+1) .
\end{array}
$$

The following distinct values for the unknowns may be chosen:

$$
s_{1}=0, t_{1}=1, s_{3}=1-y \text { and } t_{3}=y \text {. }
$$

Thus the following theorem is obtained.

THEOREM 8.1. Using the method of sum composition it is possible to construct a set $\mathrm{O}\left(p^{\alpha}+4,2\right)$ for all primes $p$ of the form $4 m+1$ or $8 m+3, p$ $\geq 11$.

Corollary 8.1. The composed $\mathrm{O}\left(p^{\alpha}+4,2\right)$ has at least 3 common parallel transversals if $p=11$ and at least 4 common parallel transversals if $p$ $>11$.

Proof. The original $\mathrm{O}\left(p^{\alpha}, 2\right)$ has $p^{\alpha}$ common parallel transversals. Since $p^{\alpha} \geq 11$, after removing 8 common parallel transversals there remain at least 3 common parallel transversals in the corresponding portion of $\mathrm{O}\left(p^{\alpha}, 2\right)$ in the composed $\mathrm{O}\left(p^{\alpha}+4,2\right)$. Now it is known that any $\mathrm{O}(4,2)$ has 4 common parallel transversals. Thus any $t \leq 4$ common parallel transversals of the corner $\mathrm{O}(4,2)$ in the composed set along with any $t$ common parallel transversals in the portion corresponding to $\mathrm{O}\left(p^{\alpha}, 2\right)$ form three common parallel transversals for the entire set.

EXAMPle 8.1. By letting $p=11$ one can construct an $\mathrm{O}(15,2)$ via sum composition of an $\mathrm{O}(11,2)$ and an $\mathrm{O}(4,2)$. Since $p=11$ falls in Case 1 , then $y=8, x=7, S=\{9,8,3,2\}$ and $T=\{0,5,1,6\}$. Utilizing the projection rules given in Case 1 the following $\mathrm{O}(15,2)$ is obtained. 


\begin{tabular}{rrrrrrrrrrr|rrrr}
0 & 1 & $D$ & $C$ & 4 & 5 & 6 & 7 & $B$ & $A$ & 10 & 9 & 8 & 3 & 2 \\
7 & $D$ & $C$ & 10 & 0 & 1 & 2 & $B$ & $A$ & 5 & 6 & 4 & 3 & 9 & 8 \\
$D$ & $C$ & 5 & 6 & 7 & 8 & $B$ & $A$ & 0 & 1 & 2 & 10 & 9 & 4 & 3 \\
$C$ & 0 & 1 & 2 & 3 & $B$ & $A$ & 6 & 7 & 8 & $D$ & 5 & 4 & 10 & 9 \\
6 & 7 & 8 & 9 & $B$ & $A$ & 1 & 2 & 3 & $D$ & $C$ & 0 & 10 & 5 & 4 \\
2 & 3 & 4 & $B$ & $A$ & 7 & 8 & 9 & $D$ & $C$ & 1 & 6 & 5 & 0 & 10 \\
9 & 10 & $B$ & $A$ & 2 & 3 & 4 & $D$ & $C$ & 7 & 8 & 1 & 0 & 6 & 5 \\
5 & $B$ & $A$ & 8 & 9 & 10 & $D$ & $C$ & 2 & 3 & 4 & 7 & 6 & 1 & 0 \\
$B$ & $A$ & 3 & 4 & 5 & $D$ & $C$ & 8 & 9 & 10 & 0 & 2 & 1 & 7 & 6 \\
$A$ & 9 & 10 & 0 & $D$ & $C$ & 3 & 4 & 5 & 6 & $B$ & 8 & 7 & 2 & 1 \\
4 & 5 & 6 & $D$ & $C$ & 9 & 10 & 0 & 1 & $B$ & $A$ & 3 & 2 & 8 & 7 \\
\hline 8 & 2 & 7 & 1 & 6 & 0 & 5 & 10 & 4 & 9 & 3 & $A$ & $B$ & $C$ & $D$ \\
1 & 6 & 0 & 5 & 10 & 4 & 9 & 3 & 8 & 2 & 7 & $B$ & $A$ & $D$ & $C$ \\
10 & 4 & 9 & 3 & 8 & 2 & 7 & 1 & 6 & 0 & 5 & $C$ & $D$ & $A$ & $B$ \\
3 & 8 & 2 & 7 & 1 & 6 & 0 & 5 & 10 & 4 & 9 & $D$ & $C$ & $B$ & $A$
\end{tabular}

\begin{tabular}{rrrrrrrrrrr|rrrr}
$D$ & $C$ & 2 & 3 & 4 & $B$ & $A$ & 7 & 8 & 9 & 10 & 6 & 1 & 5 & 0 \\
$C$ & 9 & 10 & 0 & $B$ & $A$ & 3 & 4 & 5 & 6 & $D$ & 2 & 8 & 1 & 7 \\
5 & 6 & 7 & $B$ & $A$ & 10 & 0 & 1 & 2 & $D$ & $C$ & 9 & 4 & 8 & 3 \\
2 & 3 & $B$ & $A$ & 6 & 7 & 8 & 9 & $D$ & $C$ & 1 & 5 & 0 & 4 & 10 \\
10 & $B$ & $A$ & 2 & 3 & 4 & 5 & $D$ & $C$ & 8 & 9 & 1 & 7 & 0 & 6 \\
$B$ & $A$ & 9 & 10 & 0 & 1 & $D$ & $C$ & 4 & 5 & 6 & 8 & 3 & 7 & 2 \\
$A$ & 5 & 6 & 7 & 8 & $D$ & $C$ & 0 & 1 & 2 & $B$ & 4 & 10 & 3 & 9 \\
1 & 2 & 3 & 4 & $D$ & $C$ & 7 & 8 & 9 & $B$ & $A$ & 0 & 6 & 10 & 5 \\
9 & 10 & 0 & $D$ & $C$ & 3 & 4 & 5 & $B$ & $A$ & 8 & 7 & 2 & 6 & 1 \\
6 & 7 & $D$ & $C$ & 10 & 0 & 1 & $B$ & $A$ & 4 & 5 & 3 & 9 & 2 & 8 \\
3 & $D$ & $C$ & 6 & 7 & 8 & $B$ & $A$ & 0 & 1 & 2 & 10 & 5 & 9 & 4 \\
\hline 0 & 4 & 8 & 1 & 5 & 9 & 2 & 6 & 10 & 3 & 7 & $A$ & $B$ & $C$ & $D$ \\
7 & 0 & 4 & 8 & 1 & 5 & 9 & 2 & 6 & 10 & 3 & $D$ & $C$ & $B$ & $A$ \\
8 & 1 & 5 & 9 & 2 & 6 & 10 & 3 & 7 & 0 & 4 & $B$ & $A$ & $D$ & $C$ \\
4 & 8 & 1 & 5 & 9 & 2 & 6 & 10 & 3 & 7 & 0 & $C$ & $D$ & $A$ & $B$
\end{tabular}

Example 8.2. Considering Case 2 and letting $p=13$ one can construct an $\mathrm{O}(17,2)$ by sum composition of an $\mathrm{O}(13,2)$ and an $\mathrm{O}(4,2)$. In this case $y=5, x=8, S=\{0,11,9,4\}$, and $T=\{1,3,5,10\}$. The detail of construction is left to the reader.

REMARK 8.1. Utilizing a different pattern than those considered here, Ruiz and Seiden [9] have constructed a family of $O\left(n_{1}+4,2\right), n_{1}=1,2$, $3(\bmod 7)$ using $x=2$ and $y=5 \pm \sqrt{-7}) / 4$. One can show that the same result can be obtained by starting with their pattern and assuming that $x y$ $=1$. 
9. Construction of a family of $\mathrm{O}\left(n_{1}+5,2\right)$. Consider the sum composition of $\mathrm{O}\left(p^{\alpha}, 2\right)$ based on $\mathrm{GF}\left(p^{\alpha}\right), p \geq 11$ and on $\mathrm{O}(5,2)$ based on a system of projections and capturing of the lost transversals given by

$$
\begin{array}{ll}
k_{v}\left(s_{1}, t_{1}\right)=t_{5} & k_{h}\left(s_{1}, t_{1}\right)=s_{2} \\
k_{v}\left(s_{2}, t_{2}\right)=t_{1} & k_{h}\left(s_{2}, t_{2}\right)=s_{3} \\
k_{v}\left(s_{3}, t_{3}\right)=t_{2} & k_{h}\left(s_{3}, t_{3}\right)=s_{4} \\
k_{v}\left(s_{4}, t_{4}\right)=t_{3} & k_{h}\left(s_{4}, t_{4}\right)=s_{5} \\
k_{v}\left(s_{5}, t_{5}\right)=s_{1} & k_{h}\left(s_{5}, t_{5}\right)=t_{4} .
\end{array}
$$

It can be shown that this system has a solution with distinct components only if $x y=1$. Otherwise $\Sigma s_{i}=\Sigma t_{i}$ implies $s_{1}=t_{4}$. Using the condition $x y$ $=1$ this system will yield a solution with distinct components only if $y^{2}+4$ $=0$ in $\mathrm{GF}(p)$. This implies that -1 has to be a quadratic residue in $\mathrm{GF}(p)$, i.e. $p$ has to be of the form $4 m+1$. This system of equations yields the values for $s_{i}$ and $t_{i}, i=2,3,4,5$ in terms of $s_{1}$ and $t_{1}$ that may be expressed as

$$
\begin{array}{ll}
s_{2}=\left[y s_{1}+t_{1}\right] /(y+1) & t_{2}=\left[(y+2) t_{1}-s_{1}\right] /(y+1) \\
s_{3}=\left[(y-1) s_{1}+2 t_{1}\right] /(y+1) & t_{3}=\left[(y+3) t_{1}-2 s_{1}\right] /(y+1) \\
s_{4}=\left[(y-2) s_{1}+3 t_{1}\right] /(y+1) & t_{4}=\left[(y+4) t_{1}-3 s_{1}\right] /(y+1) \\
s_{5}=\left[(y-3) s_{1}+4 t_{1}\right] /(y+1) & t_{5}=\left[y t_{1}+s_{1}\right] /(y+1) .
\end{array}
$$

For the choice of $s_{1}=0$ and $t_{1}=1$ the remaining components become

$$
\begin{aligned}
& s_{2}=1 /(y+1) \\
& s_{3}=2 /(y+1) \\
& s_{4}=3 /(y+1) \\
& s_{5}=4 /(y+1)
\end{aligned}
$$

$$
\begin{aligned}
& t_{2}=(y+2) /(y+1) \\
& t_{3}=(y+3) /(y+1) \\
& t_{4}=(y+4) /(y+1) \\
& t_{5}=y /(y+1) .
\end{aligned}
$$

It is easy to check that the ten values for the unknowns are distinct except for $p=13$, the smallest prime under consideration. Clearly the values of the sets $S$ and $T$ separately are distinct. As to the differences between the elements of the set $S$ and $T$

$$
s_{5}-t_{1}=s_{4}-t_{5}=s_{2}-s_{4}=0
$$

when $y^{2}=9$ or $13=0$. For $p=13$ one may use the example given in [8] with $x=2, y=7, S=\{0,1,2,3,4\}$ and $T=\{10,11,12,13,9\}$. In this particular case the transversal $s_{i}$ should be projected on the $(13+i)$ th row and column in the order written above, and similarly for the transversal $t_{i}$. Thus the following theorem is established. 
THEOREM 9.1. Using the method of sum composition it is possible to construct a set $\mathrm{O}\left(p^{\alpha}+5,2\right)$ for all $p$ of the form $4 m+1, p \geq 13$.

Note that the order of the composed Latin squares in Theorem 9.1 is of the form $4 t+2$.

Corollary 9.1. The composed $\mathrm{O}\left(p^{\alpha}+5,2\right)$ has at least 3 common parallel transversals if $p=13$ and at least 5 common parallel transversals if $p$ $>13$.

The proof is analogous to the proof of Corollary 8.1 .

EXAMPLE 9.1. Using the method of Theorem 9.1 a pair of orthogonal Latin squares of order $18=13+5$ and a pair of order $22=17+5$ can be constructed. For $p=13, x, y, S$ and $T$ are as above. For $p=17$

$$
x=2, y=9, S=\{0,12,7,2,14\} \text { and } T=\{1,13,8,3,6\} \text {. }
$$

The exhibition of these squares is left to the reader.

REMARK 9.1. For $n=n_{1}+n_{2} \leq 100$ and of the form $4 t+2$ there are two instances where we can construct an $\mathrm{O}(n, 2)$ by either Theorem 7.1 or Theorem 9.1. They are 22 and 94, which can be decomposed in two different ways,

$$
\begin{aligned}
& 22=19+3 \text { or } 17+5 \\
& 94=91+3 \text { or } 89+5
\end{aligned}
$$

10. Continuation of research on the method of sum composition. The results obtained in this paper suggest at least two directions for the continuation of this research.

For the first direction the results of this paper are considered as a first step in exploring the problem of construction of Latin squares and orthogonal Latin squares via sum composition. It seems obvious that investigating the problem of construction of orthogonal Latin squares for increased values of $n_{2}$ will become overwhelming and uninspiring unless a new method of attack can be found. One possibility may be to choose an especially symmetric pattern which could be generalized to some sets of values of $n_{2}$, say, of specific structure. It seems plausible that for a fixed $n_{2}$, one pattern could do for all primes conveniently changing the value of the 
function $(1-x) /(x-y)$. This amounts to giving up the assumption $x y=$ 1. In fact that was done in order to complete the case $\mathrm{O}\left(n_{1}+3,2\right)$ for all primes.

There is another important reason to consider cases for which $x y \neq 1$. $\mathrm{O}(n, t)$ sets with $t>2$ must allow cases of $\mathrm{O}(n, 2)$ constructed under the assumption $x y \neq 1$. In such cases the assumption $\Sigma s_{i}=\Sigma t_{i}=c$ must substitute the equality $x y=1$. It is possible that one could enumerate the solutions of $\mathrm{O}(n, 2)$ as a function of $c$. This would enable an exhaustive search for mutually orthogonal Latin squares with or without the assumption $x y=1$. It may be worthwhile to illustrate this idea by an example considered in [8]. There an exhaustive search was made for all sets of $\mathrm{O}(7+$ $3,2)$ with $S=\{0,1,3\}, T=\{2,4,5\}$ with or without the condition $x y=1$. In this case $\Sigma s_{i}=\Sigma t_{i}=4(\bmod 7)$. It is easy to show that for each of the distinct elements of $\mathrm{GF}(7)$ there is just one available pair of $\{S, T\}$ for consideration. Hence, in total, consideration of seven pairs exhausts all possible cases. For $p=7$ all the sets are difference sets. The question now arises: for which fields does this property hold, which could reduce the search even further?

Concerning the second direction the following remarks appear suggestive. If $B(x)$ and $B(y), x=y^{-1}$ based on Galois field $\mathrm{GF}(n)$ form an $\mathrm{O}(n, 2)$, then it is impossible to construct an $\mathrm{O}(n+1,2)$ by sum composition of this $\mathrm{O}(n, 2)$ and a trivial pair of orthogonal Latin squares of order unity. This forces $S$ to be equal to $T$, which can be seen by the fact that $S$ and $T$ each contain only one element, say $s$ and $t$, respectively. Now $k_{v}(s, t)$ $=(y t+s)(1+y)^{-1}$ will be equal to $s$ or $t$ only if $t=s$, but we require $S \cap$ $T=\phi$. However, with some modifications of the method of sum composition this can be done. Consider the following example:

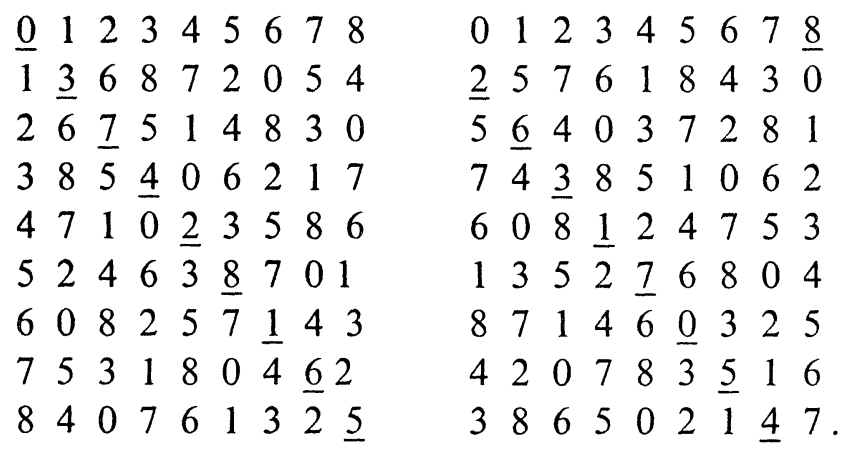

These two Latin squares of order 9 are obviously not orthogonal. However, all the cells on the main diagonals, or parallel to the main 
diagonals, form common parallel transversals. Now project the underlined transversal in each square on the tenth row and column and replace the corresponding cells with 9 . Finally, add a $1 \times 1$ Latin square in the lower right corner to obtain

\begin{tabular}{lllllllll|l}
9 & 1 & 2 & 3 & 4 & 5 & 6 & 7 & 8 & 0 \\
1 & 9 & 6 & 8 & 7 & 2 & 0 & 5 & 4 & 3 \\
2 & 6 & 9 & 5 & 1 & 4 & 8 & 3 & 0 & 7 \\
3 & 8 & 5 & 9 & 0 & 6 & 2 & 1 & 7 & 4 \\
4 & 7 & 1 & 0 & 9 & 3 & 5 & 8 & 6 & 2 \\
5 & 2 & 4 & 6 & 3 & 9 & 7 & 0 & 1 & 8 \\
6 & 0 & 8 & 2 & 5 & 7 & 9 & 4 & 3 & 1 \\
7 & 5 & 3 & 1 & 8 & 0 & 4 & 9 & 2 & 6 \\
8 & 4 & 0 & 7 & 6 & 1 & 3 & 2 & 9 & 5 \\
\hline 0 & 3 & 7 & 4 & 2 & 8 & 1 & 6 & 5 & 9
\end{tabular}

\begin{tabular}{lllllllll|l}
0 & 1 & 2 & 3 & 4 & 5 & 6 & 7 & 8 & 9 \\
9 & 5 & 7 & 6 & 1 & 8 & 4 & 3 & 0 & 2 \\
5 & 9 & 4 & 0 & 3 & 7 & 2 & 8 & 1 & 6 \\
7 & 4 & 9 & 8 & 5 & 1 & 0 & 6 & 2 & 3 \\
6 & 0 & 8 & 9 & 2 & 4 & 7 & 5 & 3 & 1 \\
1 & 3 & 5 & 2 & 9 & 6 & 8 & 0 & 4 & 7 \\
8 & 7 & 1 & 4 & 6 & 9 & 3 & 2 & 5 & 0 \\
4 & 2 & 0 & 7 & 8 & 3 & 9 & 1 & 6 & 5 \\
3 & 8 & 6 & 5 & 0 & 2 & 1 & 9 & 7 & 4 \\
\hline 2 & 6 & 3 & 1 & 7 & 0 & 5 & 4 & 8 & 9
\end{tabular}

The reader can check for himself that these Latin squares of order 10 are orthogonal. Note that these two orthogonal Latin squares have many common transversals all sharing the lower right corner cell. These common transversals can be located on the diagonals parallel to the main diagonal. It is easy to show that this $O(10,2)$ is not isomorphic with our previous $\mathrm{O}(10,2)$ derived by composition of an $\mathrm{O}(7,2)$ and an $\mathrm{O}(3,2)$.

The preceding example indicates a possible modification of sum composition method, viz, starting with non-orthogonal Latin squares. But of course they should have certain combinatorial properties and this matter is under investigation.

Before closing this section note that sum composition with Latin squares of order unity has two important consequences. First, there is no bound on the number of mutually orthogonal Latin squares of order unity. Second, in the process of sum composition only two common parallel transversals get lost for each composition. These characteristics are very important if one hopes to construct a set consisting of more than two orthogonal Latin squares by the sum composition method.

We wish to thank Mr. W. Allard for searching, with the aid of a high speed computer, for roots of fourth degree equations in some $\operatorname{GF}(p)$. 


\section{REFERENCES}

1. R. C. Bose, S. S. Shrikhande and E. T. Parker, Further results on the construction of mutually orthogonal Latin squares and the falsity of Euler's conjecture, Canad. J. Math., 12 (1960), 189-203.

2. W. T. Federer, Construction of classes of experimental designs using transversals in Latin square and Hedayat's sum composition method, in Statistical Papers in Honor of G. W. Snedecor (edited by T. A. Bancroft), (1972), 91-114, Iowa State University Press.

3. A. Hedayat, An application of sum composition: Aself orthogonal Latin square of order 10, J. Combinatorial Theory, Series A, 14 (1973), 256-260.

4. A. Hedayat and E. Seiden, On a method of sum composition of orthogonal Latin squares, RM-238 (1969), Department of Statistics and Probability, Michigan State University.

5. , On a method of sum composition of orthogonal Latin squares II, RM-257 (1970), Department of Statistics and Probability, Michigan State University.

6. _ On a method of sum composition of orthogonal Latin squares III, RM-259 (1970), Department of Statistics and Probability, Michigan State University.

7. A. Hedayat, E. T. Parker and W. T. Federer, The existence and construction of two families of designs for two successive experiments, Biometrika, 57 (1970), 351-356.

8. A. Hedayat and E. Seiden, On a method of sum composition of orthogonal Latin squares, Proceedings of the Conference on Combinatorial Geometry and Its Applications, Perugia, Italy, September 11-17, 1970, 239-256.

9. F. Ruiz and E. Seiden, Some results on the construction of orthogonal Latin squares by the method of sum composition, J. Combinatorial Theory, Series A, 16 (1974), 230-240.

Received December 18, 1972 and in revised form September 20, 1974. (Research was supported in part by 5R01-GM-05900 at Cornell University, and AFOSR73-2527A at the Florida State University.

University of Illinois at Chicago Circle AND

Michigan State University 



\section{PACIFIC JOURNAL OF MATHEMATICS}

\section{EDITORS}

RICHARD ARENS (Managing Editor)

University of California

Los Angeles, California, 90024

\section{R. A. BEAUMONT}

University of Washington

Seattle, Washington 98105

\section{J. DugunDJI}

Department of Mathematics University of Southern California Los Angeles, California 90007

D. Gillbarg AND J. MilgRAM

Stanford University Stanford, California 94305

\section{ASSOCIATE EDITORS}

E. F. BECKENBACH

B. H. NEUMANN

SUPPORTING

UNIVERSITY OF BRITISH COLUMBIA

UNIVERSITY OF CALIFORNIA

MONTANA STATE UNIVERSITY

UNIVERSITY OF NEVADA

NEW MEXICO STATE UNIVERSITY

OREGON STATE UNIVERSITY

UNIVERSITY OF OREGON OSAKA UNIVERSITY
F. WOLF

K. YosHIDA

INSTITUTIONS

UNIVERSITY OF SOUTHERN CALIFORNIA

STANFORD UNIVERSITY

UNIVERSITY OF TOKYO

UNIVERSITY OF UTAH

WASHINGTON STATE UNIVERSITY

UNIVERSITY OF WASHINGTON

AMERICAN MATHEMATICAL SOCIETY

NAVAL WEAPONS CENTER

The Supporting Institutions listed above contribute to the cost of publication of this Journal, but they are not owners or publishers and have no responsibility for its content or policies.

Mathematical papers intended for publication in the Pacific Journal of Mathematics should be in typed form or offset-reproduced, (not dittoed), double spaced with large margins. Underline Greek letters in red, German in green, and script in blue. The first paragraph or two must be capable of being used separately as a synopsis of the entire paper. Items of the bibliography should not be cited there unless absolutely necessary, in which case they must be identified by author and Journal, rather than by item number. Manuscripts, in duplicate if possible, may be sent to any one of the five editors. Please classify according to the scheme of Math. Rev. Index to Vol. ${ }^{39}$. All other communications to the editors should be addressed to the managing editor, or Elaine Barth, University of California, Los Angeles, California, 90024.

100 reprints are provided free for each article, only if page charges have been substantially paid. Additional copies may be obtained at cost in multiples of 50 .

The Pacific Journal of Mathematics is issued monthly as of January 1966. Regular subscription rate: $\$ 72.00$ a year (6 Vols., 12 issues). Special rate: $\$ 36.00$ a year to individual members of supporting institutions.

Subscriptions, orders for back numbers, and changes of address should be sent to Pacific Journal of Mathematics, 103 Highland Boulevard, Berkeley, California 90708.

\section{PUBLISHED BY PACIFIC JOURNAL OF MATHEMATICS, A NON-PROFIT CORPORATION}

Copyright (C) 1974 by Pacific Journal of Mathematics

Manufactured and first issued in the U.S.A. 


\section{Pacific Journal of Mathematics}

\section{Vol. 54, No. $2 \quad$ June, 1974}

John Edward Coury, Walsh series with coefficients tending monotonically to

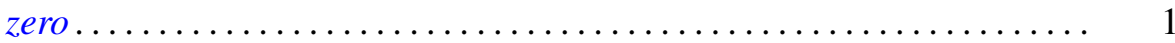

Patrick Michael Fitzpatrick and Walter Volodymyr Petryshyn, Fixed point theorems for multivalued noncompact acyclic mappings ............

Irving Leonard Glicksberg, More on Phragmén-Lindelöf for function

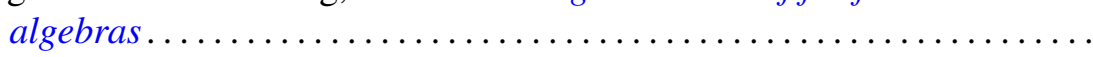

Adilson Goncalves, Structural constants. II .................. 39

Richard P. Gosselin, Closure theorems for affine transformation groups .... 53

Ralph Peter Grimaldi, Baer and UT-modules over domains ........... 59

Edward Grossman, On the prime ideal divisors of $\left(a^{n}-b^{n}\right) \ldots \ldots \ldots \ldots . \ldots 73$

A. Hedayat and Ester Seiden, On the theory and application of sum composition of Latin squares and orthogonal Latin squares.......... .

Gerald L. Itzkowitz, Continuous measures, Baire category, and uniform continuity in topological groups ......................... 115

Francis Masat, Right simple congruences on a semigroup ............ 127

Robert Harvey Oehmke, Right congruences and semisimplicity for Rees matrix semigroups..................................

Qazi Ibadur Rahman and Jan Stankiewicz, Differential inequalities and local valency . . . . . . . . . . . . . . . . . . . . . . . . . . . . . . . . . . . . . 165

William John Reed, Random points in a simplex ................ 183

Mohan S. Shrikhande, Strongly regular graphs and group divisible

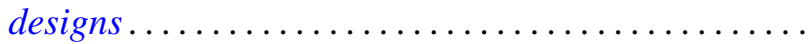

Zahava Shmuely, The structure of Galois connections ... . .

Robert C. Shock, Dual generalizations of the Artinian and Noetherian

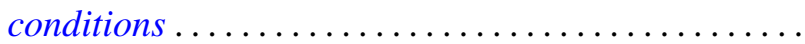

Arne Stray, Approximation and interpolation for some spaces of analytic functions in the unit disc ........................

Eldon Jon Vought, Monotone decompositions into trees of Hausdorff continua irreducible about a finite subset ............

James Wirth, The mapping cylinder axiom for WCHP fibrations ... 263

Gordon S. Woodward, Invariant means and ergodic sets in Fourier analysis... 